\title{
Insulin autoimmune syndrome in a patient with ANCA-associated glomerulonephritis
}

\author{
Tsuneyoshi $\mathrm{Ka}{ }^{1}$ \\ Yuji Moriwaki' \\ Takeshi Nakanishi ${ }^{2}$ \\ Tetsuya Yamamoto' \\ Divisions of 'Endocrinology and \\ Metabolism, and ${ }^{2}$ Kidney and Dialysis, \\ Department of Internal Medicine, \\ Hyogo College of Medicine, Hyogo, \\ Japan
}

\begin{abstract}
The patient, a 66-year-old woman, who has been under hemodialysis due to antineutrophil cytoplasm autoantibody (ANCA)-associated glomerulonephritis since 2003, was hospitalized because of cold sweating, general fatigue, and somnolence. Hypoglycemia (43 mg/dL) with markedly elevated insulin level $(1410 \mu \mathrm{U} / \mathrm{mL})$ and insulin antibody was found in her serum. She was diagnosed as having insulin autoimmune syndrome. She has not taken any medication that might cause insulin autoimmune syndrome. The possible association of ANCA-associated glomerulonephritis with insulin autoimmune syndrome is discussed.
\end{abstract}

Keywords: hypoglycemia, insulin antibody, ANCA, insulin autoimmune syndrome

Although insulin autoimmune syndrome is a relatively rare cause of hypoglycemia, it is the third leading cause of spontaneous hypoglycemia in Japan and more than 200 cases have been reported (Uchigata and Hirata 1999). This syndrome is characterized by hypoglycemic episodes, elevated insulin levels, and positive insulin antibodies in patients with no prior insulin administration (Uchigata and Hirata 1999). The cause is not fully understood, however, various drugs as well as other autoimmune disorders are considered to be involved with its development. Herein, we report the first known case of insulin autoimmune syndrome due to antineutrophil cytoplasm autoantibody (ANCA)-associated glomerulonephritis, which developed during hemodialysis.

A 66-year-old woman experienced excessive sweating, general fatigue, and somnolence was transferred to our hospital for examinations. She had a history of ANCAassociated glomerulonephritis for 5 years, and had been receiving corticosteroid and hemodialysis. The patient also had pneumothorax for the previous 3 years and multiple peptic ulcers for the previous 4 years. She had not regularly taken any medication or supplements, and had not received insulin therapy in the past. A physical examination revealed a well-nourished woman, whose consciousness level was II-10 (Japan Coma Scale). She was $148 \mathrm{~cm}$ tall and weighed $55 \mathrm{~kg}$, and her body mass index was $25 \mathrm{~kg} / \mathrm{m}^{2}$. There was no edema in the extremities, and her skin was warm and moist. Blood pressure was 117/84 $\mathrm{mmHg}$, heart rate was 96 and regular, and temperature was $36.4^{\circ} \mathrm{C}$. Her head, eyes, nose, and throat appeared to be normal, while the lungs were clear and a cardiac examination was normal. The abdomen was soft, without tenderness, organomegaly, or apparent masses.

Results of laboratory studies showed hematocrit at $34.9 \%$ and white blood cells

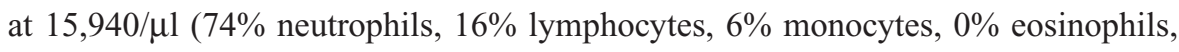
$0 \%$ basophils). Plasma glucose was $42 \mathrm{mg} / \mathrm{dl}$, and insulin and C-peptide immunoreactivity were $1410 \mu \mathrm{U} / \mathrm{m}$ and $28.8 \mathrm{ng} / \mathrm{mL}$, respectively (Fajan's index, 24.3), while serum creatinine was $6.4 \mathrm{mg} / \mathrm{dl}$ and blood urea nitrogen $26 \mathrm{mg} / \mathrm{dl}$. The calculated glomerular filtration rate was $4.83 \mathrm{~mL} / \mathrm{min}$. Serum sodium, potassium, and chloride were $140 \mathrm{mmol} / 1,4.3 \mathrm{mmol} / 1$, and $100 \mathrm{mmol} / 1$, respectively. Results of liver function, 
creatine phosphokinase, chest X-ray, and electrocardiogram examinations were unremarkable, except for elevated alkaline phosphatase at $489 \mathrm{U} / \mathrm{L}$. C-reactive protein was 7.1 $\mathrm{mg} / \mathrm{dL}$ and perinuclear-ANCA was $70.5 \mathrm{U} / \mathrm{mL}$. Rheumatoid factor and dsDNA antibody were negative. The patient had recurrent hypoglycemic bouts, therefore continuous glucose infusion was initiated. Computed tomography of the abdomen and pelvis, as well as ultrasound findings were negative for insulinoma. Antibodies to insulin were detected (insulin binding ratio $92.6 \%$, free insulin $1410 \mu \mathrm{U} / \mathrm{ml}$ ). A Scatchard plot analysis revealed that the affinity of the antibodies was low and their binding activity high, which are common characteristics of anti-insulin autoantibodies observed in insulin autoimmune syndrome (Eguchi 1989; Eguchi et al 1994; Furukawa et al 2006). Serum levels of thyroid hormone, adrenocorticotropic hormone, and glucocorticoid were normal. These findings, including the symptoms and laboratory, endocrinological, and imaging results led us to a diagnosis of insulin autoimmune syndrome.

The association between sulfhydryl compounds and development of insulin autoimmune syndrome is well known, however, the present patient had not taken sulfhydryl compounds, such as methimazole, $\alpha$-mercaptopropionyl glycine, glutathione, or captopril, which cause the production of anti-insulin antibodies. Furthermore, the HLA-DRB1*0406 allele confers a high level of susceptibility to the syndrome (Uchigata et al 1992, 1993), however, HLA analysis could not be performed in the present case, since the patient died suddenly during hemodialysis.

To our knowledge, this is the first case of insulin autoimmune syndrome complicated with ANCA-associated glomerulonephritis to be reported following the initial description of the syndrome in 1970 (Hirata et al 1970). Although the present association with ANCA-associated glomerulonephritis may have been coincidental, patients with insulin autoimmune syndrome may also have a history of other autoimmune diseases, such as Graves' disease, rheumatoid arthritis, or systemic lupus erythematous (Uchigata and Hirata 1999; Burch et al 1992). Therefore, it may be appropriate to include ANCA-associated glomerulonephritis in the group of underlying diseases of insulin autoimmune syndrome, though the relationship between ANCA and the anti-insulin antibody has not been clarified.

\section{Disclosure}

The authors report no conflicts of interest in this work.

\section{References}

Burch HB, Clement S, Sokol MS, et al. 1992. Reactive hypoglycemic coma due to insulin autoimmune syndrome: case report and literature review. Am J Med, 92:681-5.

Eguchi Y, Uchigata Y, Yao K, et al. 1994. Longitudinal changes of serum insulin concentration and insulin antibody features in persistent insulin autoimmune syndrome (Hirata's disease). Autoimmunity, 19:279-84.

Eguchi Y. 1989. Scatchard analysis of insulin autoantibodies in the insulin autoimmune syndrome. J Tokyo Wom Med Univ, 59:1286-305.

Furukawa N, Miyamura N, Nishida K, et al. 2006. Possible relevance of alpha lipoic acid contained in a health supplement in a case of insulin autoimmune syndrome. Diabetes Res Clin Pract, 75:366-7.

Hirata Y, Ishizu H, Ouchi N, et al. 1970. Insulin autoimmunity in a case with spontaneous hypoglycaemia. J Jpn Diabetes Soc, 13:312-20.

Uchigata Y, Hirata Y. 1999. Insulin autoimmune syndrome (IAS, Hirata disease). Ann Med Interne (Paris), 150:245-53.

Uchigata Y, Kuwata S, Tokunaga K, et al. 1992. Strong association of insulin autoimmune syndrome with HLA-DR4. Lancet, 339:393-4.

Uchigata Y, Kuwata S, Tsushima T, et al. 1993. Patients with Graves' disease who developed insulin autoimmune syndrome (Hirata disease) possess HLA-Bw62/Cw4/DR4 carrying DRB1*0406. J Clin Endocrinol Metab, 77:249-54. 